\title{
CONVOLUTION ESTIMATES FOR SOME MEASURES ON CURVES
}

\author{
DANIEL M. OBERLIN
}

\begin{abstract}
Suppose that $\lambda$ is a smooth measure on a curve in $R^{3}$. It is shown that $\lambda * L^{p}\left(R^{3}\right) \subset L^{q}\left(R^{3}\right)$ under certain conditions on $\lambda, p$, and $q$.
\end{abstract}

For $1 \leq p \leq \infty$, let $L^{p}$ be the usual Lebesgue space formed with respect to Lebesgue measure on $R^{n}$. It is well known that every complex Borel measure $\lambda$ on $R^{n}$ acts as a convolution operator on $L^{p}: \lambda * L^{p} \subset L^{p}$. If $\lambda$ is absolutely continuous with density in $L^{r}$ for some $r>1$, Young's inequality shows that for $1 \leq p<\infty$ we have $\lambda * L^{p} \subset L^{q}$ for some $q=q(p)>p$. One might say that such a measure is " $L^{p}$ improving." If $\lambda$ is singular, it is still possible for $\lambda$ to be $L^{p}$-improving when $p>1$. The Cantor-Lebesgue measures on $R$ are examples [1]. The purpose of this paper is to investigate similar behavior for a different class of singular measures, smooth measures supported on curves in $R^{3}$. Our motivation comes from the following two results.

THEOREM 1. Suppose $1 \leq k<n$ and $K$ is a smooth $k$-dimensional surface in $R^{n}$. Suppose that $\lambda$ is a smooth measure on $K$. If $\lambda * L^{p} \subset L^{q}$, then $(1 / p, 1 / q)$ lies in the closed triangle in $R^{2}$ with vertices $(0,0),(1,1)$, and $(n /(2 n-k),(n-k) /(2 n-k))$.

PROOF. A theorem of Hörmander [3] implies that $p \leq q$. Estimating the norms of $f$ and $\lambda * f$ when $f$ is the characteristic function of a small ball shows that $(1 / p, 1 / q)$ lies on or above the line $L$ joining $(1,1)$ and $(n /(2 n-k),(n-k) /(2 n-k))$. Since $\lambda * L^{p} \subset L^{q}$ implies $\lambda * L^{q^{\prime}} \subset L^{p^{\prime}}$, where $p^{\prime}$ and $q^{\prime}$ are the conjugate indices, $\left(1 / q^{\prime}, 1 / p^{\prime}\right)$ also lies on or above $L$. This completes the proof.

THEOREM 2. Suppose $K$ is a smooth $(n-1)$-dimensional surface in $R^{n}$ on which all $n-1$ principal curvatures are bounded away from zero. Suppose that $\lambda$ is a smooth finite measure compactly supported away from the boundary. Then $\lambda * L^{p} \subset L^{q}$ if and only if $(1 / p, 1 / q)$ lies in the closed triangle in $R^{2}$ with vertices $(0,0),(1,1)$, and $(n /(n+1), 1 /(n+1))$.

PROOF. A theorem of Littman [4] implies that $\lambda * L^{(n+1) / n} \subset L^{n+1}$. Interpolation with trivial cases completes half the proof, and Theorem 1 gives the rest.

Theorems 1 and 2 lead naturally to the conjecture that if $\lambda$ is a nice measure supported on a nice $k$-dimensional surface in $R^{n}$, then $\lambda * L^{p} \subset L^{q}$ precisely when $(1 / p, 1 / q)$ is in the closed triangle of Theorem 1 . This conjecture seems difficult even when $k=1, n=3$. Our purpose here is to prove a partial result for this case. We consider certain measures $\lambda$ on some curves in $R^{3}$ and prove that $\lambda * L^{p} \subset L^{q}$ whenever $(1 / p, 1 / q)$ lies in the triangle of Theorem 1 and on or above the line

Received by the editors October 23, 1985.

1980 Mathematics Subject Classification (1985 Revision). Primary 43A22.

Partially supported by the National Science Foundation. 
through $(1 / 2,1 / 3)$ and $(2 / 3,1 / 2)$. To show this it is sufficient, by duality and interpolation, to establish that $\lambda * L^{3 / 2} \subset L^{2}$.

THEOREM 3. Suppose that $I$ is a closed interval in $R$ and that the real-valued functions $\phi_{1}$ and $\phi_{2}$ are both polynomial functions or both trigonometric functions on I. Put $\phi(t)=\left(\phi_{1}(t), \phi_{2}(t)\right)$ and, if $j=2$ or 3 , write $\phi^{(j)}$ for the $j$ th derivative of the function $\phi$. Suppose that given any $t_{1}, t_{2} \in I$, the vectors $\phi^{(2)}\left(t_{1}\right)$ and $\phi^{(3)}\left(t_{2}\right)$ span $R^{2}$. Let $\lambda$ be the measure on $R^{3}$ defined by

$$
\langle\lambda, g\rangle=\int_{I} g\left(t, \phi_{1}(t), \phi_{2}(t)\right) d t
$$

Then $\lambda * L^{3 / 2} \subset L^{2}$.

Here are two examples where Theorem 3 applies.

EXAMPLE 1. $I=[a, b], \phi_{1}(t)=t^{2}, \phi_{2}(t)=t^{3}$.

EXAMPLE 2. $I=[0, \pi / 6], \phi_{1}(t)=\cos (t), \phi_{2}(t)=\sin (t)$.

Following are some comments on the notation to be used in the proof of Theorem 3. The symbol $f$ (resp. $g$ ) will denote an arbitrary continuous function of compact support on $R^{2}$ (resp. $R^{3}$ ). The symbol $\|\cdot\|_{p}$ will denote the norm of the indicated function in $L^{p}\left(R^{2}\right)$ or $L^{p}\left(R^{3}\right)$, whichever is appropriate. A sector $\Gamma$ in the plane is defined to be the set of all points in the plane which have a polar representation $r e^{i \theta}$ with $r \geq 0, c \leq \theta \leq d$, where $c$ and $d$ are fixed real numbers. The symbol $C$ will denote a positive constant which may increase from line to line but which depends only on the data $I$ and $\phi$ in the hypotheses of Theorem 3. Similarly, the symbol $C\left(\Gamma_{1}, \Gamma_{2}, \delta\right)$ appearing in the following lemma represents a "variable" constant which can always be chosen to depend only upon $\Gamma_{1}, \Gamma_{2}$, and $\delta$. The proof of this lemma is based on complex interpolation and follows fairly standard lines.

LEMMA. Suppose $\Gamma_{1}$ and $\Gamma_{2}$ are sectors such that $\Gamma_{2} \cap\left(\Gamma_{1} \cup-\Gamma_{1}\right)=\{0\}$. Suppose $\delta>0$. There is a positive constant $C\left(\Gamma_{1}, \Gamma_{2}, \delta\right)$ such that the following holds: Suppose $J \subset R$ is a closed interval of length not exceeding $\delta^{-1}$ and suppose that $\Psi(t)=\left(\Psi_{1}(t), \Psi_{2}(t)\right)$ is a twice differentiable function from $J$ into $R^{2}$ such that

(i) for every $t \in J: \Psi^{\prime}(t) \in \Gamma_{1}, \Psi^{(2)}(t) \in \Gamma_{2},\left|\Psi^{\prime}(t)\right| \geq \delta,\left|\Psi^{(2)}(t)\right| \geq \delta$;

(ii) for any $x \in R^{2}, J$ splits into disjoint subintervals $J_{1}, \ldots, J_{K}$ with $K \leq \delta^{-1}$ such that the scalar product $x \cdot \Psi^{(2)}(t)$ is of constant sign on each $t$-interval $J_{n}$.

The measure $\mu$ on $R^{2}$ defined by

$$
\langle\mu, f\rangle=\int_{J} f(\Psi(t)) d t
$$

satisfies

$$
\|\mu * f\|_{3} \leq C\left(\Gamma_{1}, \Gamma_{2}, \delta\right)\|f\|_{3 / 2} .
$$

PROOF. For $z \in C$ consider the distribution $d_{z}(s)=|s|^{z}$ on $R$. The map $z \rightarrow$ $d_{z}$ defines a meromorphic distribution-valued function on $C$ with simple poles at $z=-1,-3,-5, \ldots$ Thus $d_{z} /(\Gamma(z+1) / 2)$ is an entire distribution-valued function of $z$ (see [2] for details).

Now let $y_{1}$ be a unit vector orthogonal to no nonzero vector in $\Gamma_{1}$ and let $y$ be a unit vector orthogonal to $y_{1}$. 
For $z \in C$ and test functions $h$ on $R^{2}$, define the distribution $T_{z}$ by

$$
\left\langle T_{z}, h\right\rangle=\frac{1}{\Gamma((z+1) / 2)} \int_{J} \int_{-\infty}^{\infty} h(\Psi(t)+s y)|s|^{z} d s d t
$$

We will establish that

$$
\begin{aligned}
\left\|T_{i y} * h\right\|_{\infty} \leq & \left(C\left(\Gamma_{1}, \Gamma_{2}, \delta\right) / \Gamma\left(\frac{1+i y}{2}\right)\right)\|h\|_{1}, \\
\left\|T_{i y-3 / 2} * h\right\|_{2} \leq & \left(C\left(\Gamma_{1}, \Gamma_{2}, \delta\right) / \Gamma\left(\frac{3-2 i y}{4}\right)\right)\|h\|_{2}, \\
& T_{-1} * h=\mu * h
\end{aligned}
$$

Then the conclusion of the lemma will follow from the interpolation theorem in [5].

To show (1) is to show that $T_{i y}$ is an $L^{\infty}$ function of appropriate norm. The map $(s, t) \rightarrow \Psi(t)+s y$ from $R \times J$ into $R^{2}$ is one-to-one by the assumptions on $y$ and $y_{1}$ and the mean value theorem. It has Jacobian of absolute value $\left|\Psi^{\prime}(t) \cdot y_{1}\right|$, a quantity which exceeds $1 / C\left(\Gamma_{1}, \Gamma_{2}, \delta\right)$. Thus

$$
\left|\int_{J} \int_{-\infty}^{\infty} h(\Psi(t)+s y) d s d t\right| \leq C\left(\Gamma_{1}, \Gamma_{2}, \delta\right)\|h\|_{1} .
$$

This gives (1).

To obtain (2) we must estimate the Fourier transform of $T_{i y-3 / 2}$. Calculations in [2] show that the Fourier transform of $d_{z} / \Gamma((z+1) / 2)$ at $s$ is

$$
2^{z+1} \pi^{1 / 2}|s|^{-z-1} / \Gamma(-z / 2)
$$

Thus the Fourier transform of $T_{z}$ is given by

$$
\hat{T}_{z}(x)=\hat{\mu}(x) \cdot 2^{z+1} \pi^{1 / 2}|x \cdot y|^{-z-1} / \Gamma(-z / 2)
$$

(from which (3) follows). To establish (2) it is therefore sufficient to show that

$$
|x|^{1 / 2}|\hat{\mu}(x)| \leq C\left(\Gamma_{1}, \Gamma_{2}, \delta\right) \text { for } x \in R^{2} \text {. }
$$

Note that the hypotheses guarantee that

$$
\left|x \cdot \Psi^{\prime}(t)\right|+\left|x \cdot \Psi^{(2)}(t)\right| \geq|x| / C\left(\Gamma_{1}, \Gamma_{2}, \delta\right), \quad x \in R^{2}, t \in J .
$$

Now fix nonzero $x \in R^{2}$ and put $p(t)=x \cdot \Psi(t)$. By (ii), $J$ splits into disjoint subintervals $J_{1}, \ldots, J_{K}$ with $K \leq \delta^{-1}$ such that $p^{(2)}(t)$ has constant sign on each $J_{n}$. Write $\eta=|x| / 2 C\left(\Gamma_{1}, \Gamma_{2} ; \delta\right)$ and let $I_{n}^{1}=J_{n} \cap\left\{\left|p^{\prime}(t)\right|<\eta\right\}$. Since $p^{\prime}(t)$ is monotone on $J_{n}, I_{n}^{1}$ is an interval and so $J_{n} \backslash I_{n}^{1}$ is the disjoint union of $I_{n}^{2}$ and $I_{n}^{3}$, where each of $I_{n}^{2}$ and $I_{n}^{3}$ is an interval or empty. By (5) we have either $p^{(2)}(t) \geq \eta$ or $p^{(2)}(t) \leq-\eta$ on $I_{n}^{1}$. Therefore

$$
\eta^{1 / 2}\left|\int_{I_{n}^{1}} e^{-i x \cdot \Psi(t)} d t\right| \leq 4
$$

by van der Corput's lemma [7]. If $j=2$ or $j=3$ we have $p^{\prime}(t)$ monotone and either $p^{\prime}(t)>\eta$ or $p^{\prime}(t)<-\eta$ on $I_{n}^{j}$. Thus, by van der Corput's lemma again,

$$
\eta^{1 / 2}\left|\int_{I_{n}^{j}} e^{-i x \cdot \Psi(t)} d t\right| \leq \min \left\{\eta^{1 / 2} \cdot \operatorname{length}\left(I_{\eta}^{j}\right), \eta^{-1 / 2}\right\} \leq \delta^{-1 / 2}
$$


where the last inequality is because length $(J) \leq \delta^{-1}$. Adding all inequalities (6) and (7) gives (4) since there are at most $3 K \leq 3 \delta^{-1}$ such terms. This completes the proof of the lemma.

PROOF OF THEOREM 3 . Let $\tilde{\lambda}$ be defined by

$$
\langle\tilde{\lambda}, g\rangle=\int_{R^{3}} g(-x) d \lambda(x) .
$$

Since $\langle\tilde{\lambda} * \lambda * g, g\rangle=\|\lambda * g\|_{2}^{2}$, it is enough to show that

$$
\|\tilde{\lambda} * \lambda * g\|_{3} \leq C\|g\|_{3 / 2}
$$

for continuous compactly supported functions $g$ on $R^{3}$. Writing $I=[a, b]$ and $I_{u}=[\max \{a, a-u\}, \min \{b, b-u\}]$ for $|u| \leq b-a$ we have

$$
\begin{aligned}
\langle\tilde{\lambda} * \lambda, g\rangle & =\int_{a}^{b} \int_{a}^{b} g\left(t-s, \phi_{1}(t)-\phi_{1}(s), \phi_{2}(t)-\phi_{2}(s)\right) d s d t \\
& =\int_{a-b}^{b-a} \int_{I_{u}} g\left(u, \phi_{1}(s+u)-\phi_{1}(s), \phi_{2}(s+u)-\phi_{2}(s)\right) d s d u .
\end{aligned}
$$

For $|u| \leq b-a$, define the measure $\lambda_{u}$ on $R^{2}$ by

$$
\left\langle\lambda_{u}, f\right\rangle=\int_{I_{u}} f\left(\phi_{1}(s+u)-\phi_{1}(s), \phi_{2}(s+u)-\phi_{2}(s)\right) d s .
$$

We will prove that

$$
\left\|\lambda_{u} * f\right\|_{3} \leq C|u|^{-2 / 3}\|f\|_{3 / 2}
$$

Assume (9) for the moment and write $g(t, x)\left(t \in R, x \in R^{2}\right)$ for a continuous compactly supported function on $R^{3}$. Then

$$
\begin{aligned}
\|\tilde{\lambda} * \lambda * g\|_{3} & =\|\| \int_{a-b}^{b-a} \lambda_{u} * g(t-u, \cdot)(x) d u\left\|_{3, x}\right\|_{3, t} \\
& \leq\left\|\int_{a-b}^{b-a}\right\| \lambda_{u} * g(t-u, \cdot)(x)\left\|_{3, x} d u\right\|_{3, t} \\
& \leq C\left\|\int_{a-b}^{b-a}|u|^{-2 / 3}\right\| g(t-u, x)\left\|_{3 / 2, x} d u\right\|_{3, t} .
\end{aligned}
$$

By the boundedness of the Riesz potential of order $\frac{1}{3}$ as a mapping from $L^{3 / 2}(R)$ to $L^{3}(R)$ (see p. 119 of $[6]$ ), this last term is dominated by

$$
C\|\| g(t, x)\left\|_{3 / 2, x}\right\|_{3 / 2, t}=C\|g\|_{3 / 2} \text {. }
$$

Thus (8), and so Theorem 3, will be proved when (9) is established.

By the hypotheses of Theorem 3 there is an $\eta>0$ such that

$$
\left|\phi^{(2)}(t)\right|,\left|\phi^{(3)}(t)\right| \geq \eta \quad \text { if } t \in I .
$$

The sets

$$
K_{1}=\left\{\phi^{(2)}(t) /\left|\phi^{(2)}(t)\right|: t \in I\right\}, \quad K_{2}=\left\{\phi^{(3)}(t) /\left|\phi^{(3)}(t)\right|: t \in I\right\}
$$

are disjoint closed intervals on the unit circle in $R^{2}$. If $i=1$ or 2 , define $\Gamma_{i}$ to be the sector $\left\{r v: r \geq 0, v \in K_{i}\right\}$. The hypotheses of Theorem 3 imply that 
$\Gamma_{2} \cap\left(\Gamma_{1} \cup-\Gamma_{1}\right)=\{0\}$ and thus that the $\Gamma_{i}$ are convex. By (10), for $i=1$ or 2 the set $\phi^{(i+1)}(I)$ lies in a proper closed subset $\Gamma_{i}^{\prime}$ of $\Gamma_{i} \backslash\{0\}$, and $\Gamma_{i}^{\prime}$ can be chosen to be convex. Fix $\delta>0$ such that

$(\alpha)\left\{x \in R^{2}:|x|<\delta\right\} \cap \Gamma_{i}^{\prime}=\varnothing$ for $i=1,2$;

( $\beta) b-a \leq \delta^{-1}$;

( $\gamma$ ) For $u \in[a-b, b-a]$ and $x \in R^{2}, I_{u}$ splits into disjoint subintervals $J_{1}, \ldots, J_{K}$ with $K \leq \delta^{-1}$ such that $x \cdot\left(\phi^{(2)}(t+u)-\phi^{(2)}(t)\right)$ is of constant sign on each $t$-interval $J_{n}$. (This is where we use the hypothesis that $\phi_{1}$ and $\phi_{2}$ be both polynomial or trigonometric functions.)

Now fix $u \in[a-b, b-a]$. Define $\Psi(t)$ on $I_{u}$ by

$$
\Psi(t)=\frac{1}{u}(\phi(t+u)-\phi(t)) .
$$

Letting $\Gamma_{1}, \Gamma_{2}$, and $\delta$ be as above, we claim that $\Psi(t)$ satisfies the hypotheses of the lemma. The hypothesis (i) for $\Psi^{\prime}(t)$ follows from

$$
\Psi^{\prime}(t)=\frac{1}{u} \int_{t}^{t+u} \phi^{(2)}(s) d s
$$

combined with the convexity of $\Gamma_{1}^{\prime}$ and $(\alpha)$. The hypothesis (i) for $\Psi^{(2)}(t)$ follows similarly. The hypothesis (ii) is a consequence of $(\gamma)$, and the hypothesis that length $(J)$ not exceed $\delta^{-1}$ is covered by $(\beta)$. Thus it follows, with the notation of the lemma, that

$$
\|\mu * f\|_{3} \leq C\|f\|_{3 / 2} .
$$

For functions $f$ on $R^{2}$ and $u \in R$, put $D_{u} f(x)=f(u x), x \in R^{2}$. Then

$$
\lambda_{u} * f(x)=\int_{I_{u}} f(x-u \Psi(t)) d t=\int_{I_{u}} f\left(u\left(\frac{x}{u}-\Psi(t)\right)\right) d t=D_{1 / u}\left(\mu *\left(D_{u} f\right)\right)(x) .
$$

Now (9) follows from (11) and the fact that

$$
\left\|D_{u} f\right\|_{p}=|u|^{-2 / p}\|f\|_{p}
$$

\section{REFERENCES}

1. M. Christ, Convolution estimates for Cantor-Lebesgue measures, preprint.

2. I. M. Gelfand and G. E. Shilov, Generalized functions, Academic Press, New York, 1964.

3. L. Hörmander, Estimates for translation-invariant operators in $L^{p}$ spaces, Acta Math. 104 (1960), 93-140.

4. W. Littman, $L^{p}-L^{q}$ estimates for singular integral operators, Proc. Sympos. Pure Math., vol. 23, Amer. Math. Soc., Providence, R.I., 1973.

5. E. M. Stein, Interpolation of linear operators, Trans. Amer. Math. Soc. 87 (1958), 159-172.

6. __ Singular integrals and differentiability properties of functions, Princeton Univ. Press, Princeton, N.J., 1970.

7. A. Zygmund, Trigonometric series, Cambridge Univ. Press, Cambridge, 1959.

Department of Mathematics, Florida State University, Tallahassee, FLORIDA 32306-3027 